\title{
Microstructures and resistivity of cuprate/manganite bilayer deposited on $\mathrm{SrTiO}_{3}$ substrate
}

\author{
W. S. Tan, a) X. S. Wu, J. Du, J. S. Liu, A. Hu, and S. S. Jiang \\ Department of Physics, National Laboratory of Solid State Microstructures, Nanjing University, \\ Nanjing 210093, China \\ J. Wang, Z. H. Wu, W. L. Zheng, and Q. J. Jia \\ Institute of High Energy Physics, Chinese Academy of Science, Beijing 100039, China \\ J. Gao \\ Department of Physics, The University of Hong Kong, Pokfulam Road, Hong Kong, China
}

(Presented on 14 November 2002)

\begin{abstract}
Thin $\mathrm{YBa}_{2} \mathrm{Cu}_{3} \mathrm{O}_{7-\delta} / \mathrm{La}_{0.67} \mathrm{Ca}_{0.33} \mathrm{MnO}_{3}$ (YBCO/LCMO) films were grown on $\mathrm{SrTiO}_{3}(\mathrm{STO})$ substrates by magnetron sputtering technique. The microstructures of the bilayers were characterized and a standard four-probe technique was applied to measure the resistivity of the samples. The interdiffusions at the YBCO/LCMO and LCMO/STO interfaces formed two transient layers with the thickness of about 3 and $2 \mathrm{~nm}$, respectively. All the bilayers were well textured along the $c$ axis. At low temperature, the superconductivity can only be observed when the thickness of YBCO is more than $25 \mathrm{~nm}$. When the thickness of YBCO is less than $8 \mathrm{~nm}$, the bilayers show only ferromagnetism. The superconductivity and ferromagnetism perhaps coexist in the bilayer with the YBCO thickness of $12.5 \mathrm{~nm}$. These interesting properties are related to the interaction between spin polarized electrons in the manganites and the cooper pairs in the cuprates. (c) 2003 American Institute of Physics. [DOI: 10.1063/1.1541653]
\end{abstract}

\section{INTRODUCTION}

The classical proximity effect between the conventional superconductor $(S)$ and metallic ferromagnet $(F)$ thin layers has been studied in many systems theoretically and experimentally. ${ }^{1-6}$ It is now believed that the heterostructure, combined high $T_{c}$ superconductor (HTS) with colossal magnetoresistance (CMR) oxide, has potential application in fast devices with high gain, due to the almost full spin polarization of CMR oxides, together with the fast relaxation times and low carrier density of HTS. In most cases, perfect crystalline structure will play an important role in ensuring the properties of devices. Fortunately, the similarity in the structure of high $T_{c}$ cuprate and CMR manganite makes it possible to prepare high quality heterostructures. Therefore, many studies have been performed on HTS/CMR $F / S$ heterostructures, especially on cuprate/manganite heterostructures. ${ }^{7-11}$ Most of these studies are devoted to the superconductivity and magnetism of cuprate/manganite multilayers and superlattices. It is generally believed that the superconductivity is suppressed by spin-polarized carriers injected into superconductor. ${ }^{12,13}$ In addition, it is significant to point out that in Ref. 13 the value of self-injection length of spinpolarized carriers $(77 \mathrm{~K})$ was estimated to be $\sim 90 \mathrm{~nm}$ in a $\mathrm{La}_{0.7} \mathrm{Ca}_{0.3} \mathrm{MnO}_{3} / \mathrm{YBa}_{2} \mathrm{Cu}_{3} \mathrm{O}_{7-\delta}$ bilayer from the measurement of critical current density $J_{c}$.

Although many studies have been performed in cuprate/ manganite multilayers and superlattices as mentioned above, there is little work on the relationship between microstruc-

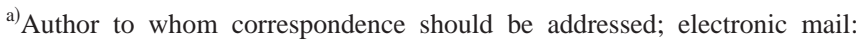
ssjiang@nju.edu.cn
}

tures and transport properties of heteroepitaxial bilayers. In this article we present the microstructure and transport properties of $\mathrm{YBa}_{2} \mathrm{Cu}_{3} \mathrm{O}_{7-\delta} / \mathrm{La}_{0.67} \mathrm{Ca}_{0.33} \mathrm{MnO}_{3}(\mathrm{YBCO} / \mathrm{LCMO})$ bilayers.

\section{EXPERIMENT}

Thin YBCO/LCMO films were grown on (100) $\mathrm{SrTiO}_{3}(\mathrm{STO})$ substrates by the off-axis rf magnetron sputtering technique. The total sputtering pressure of $25 \%$ oxygen-75\% argon mixture was kept at 10-20 Pa. The depositing temperature was $750-770^{\circ} \mathrm{C}$ for LCMO and $730-$ $750{ }^{\circ} \mathrm{C}$ for $\mathrm{YBCO}$, respectively. The growth rate for LCMO and $\mathrm{YBCO}$ was $0.6-0.7$ and $0.9-1.0 \mathrm{~nm} / \mathrm{min}$, respectively. All the samples were postannealed for $0.5 \mathrm{~h}$ at $500^{\circ} \mathrm{C}$ in 1 atm of pure oxygen. In $\mathrm{YBCO} / \mathrm{LCMO} / \mathrm{STO}$ heterostructures studied here, the LCMO thickness was fixed at $30 \mathrm{~nm}$ and the YBCO thickness $t$ for different samples are listed in Table I.

The microstructures of samples were characterized by the high resolution $\mathrm{x}$-ray diffraction, grazing incident $\mathrm{x}$-ray reflectivity (GIXR), Auger electron spectroscopy (AES), and

TABLE I. The YBCO thickness of YBCO/LCMO(30 nm)/STO $(t)$ heterostructure.

\begin{tabular}{cc}
\hline \hline Sample & $t(\mathrm{~nm})$ \\
\hline A & 50 \\
B & 25 \\
C & 12.5 \\
D & 8 \\
E & 4 \\
\hline \hline
\end{tabular}




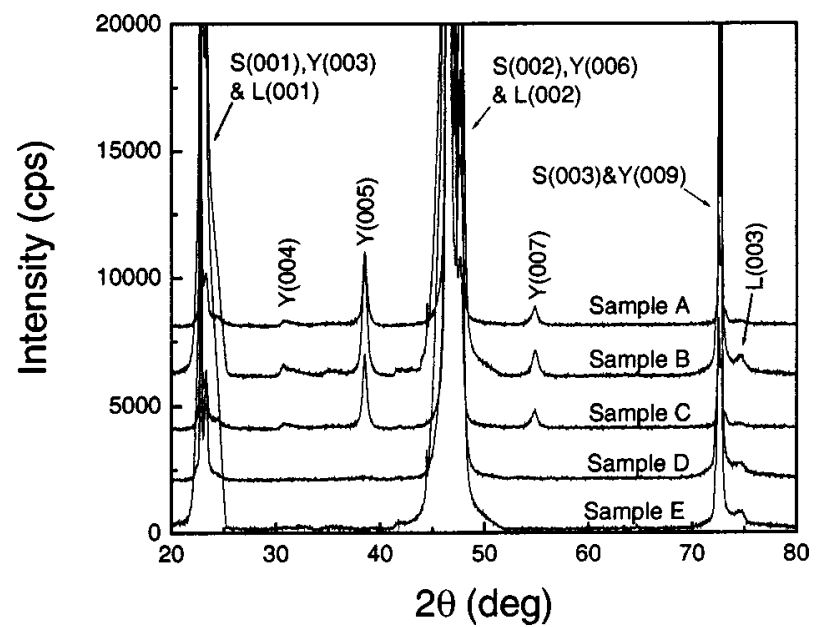

FIG. 1. High angle $\mathrm{X}$-ray diffraction patterns for samples. The capitalized letters $S, L$, and $Y$ refer to STO, LCMO, and YBCO, respectively. The numbers in parentheses is Miller indices.

atomic force microscopy (AFM). A dc four-probe method was used to measure the resistivity of the samples without magnetic field applied.

\section{RESULTS AND DISCUSSION}

Figure 1 shows high angle $\mathrm{x}$-ray diffraction pattern for samples, in which only $(00 L)$-type diffraction peaks can be found. This means that all the samples were well $c$ axis grown. From the $(00 L)$ peak position we can learn that with increasing the YBCO thickness the lattice parameter $c$ of YBCO layer decreases from 1.1744 to $1.1680 \mathrm{~nm}$, which is almost the value of that for YBCO bulk material. The inplane average parameter $a$ will increase with the increase of the thickness of YBCO. This means that the lattice of YBCO layer is partially strained (expanded) due to the lattice mismatch and finally total relaxation of strain occurs for sample A.

To investigate the microstructures at the surface and interface of film, GIXR and AFM were applied. Figure 2 shows the measured and simulated GIXR curves for samples

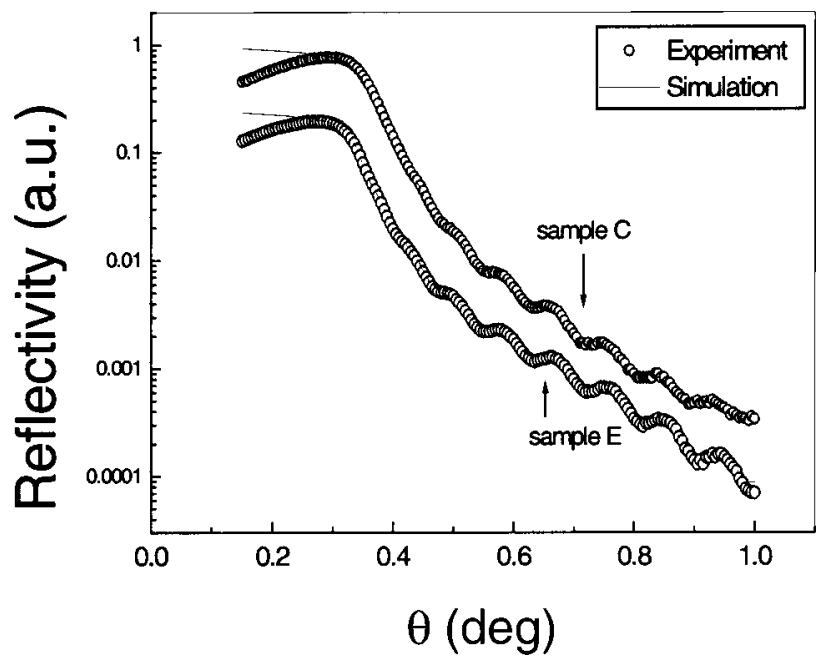

FIG. 2. The measured and simulated grazing $\mathrm{x}$-ray reflectivity profiles for samples $\mathrm{C}$ and $\mathrm{E}$.

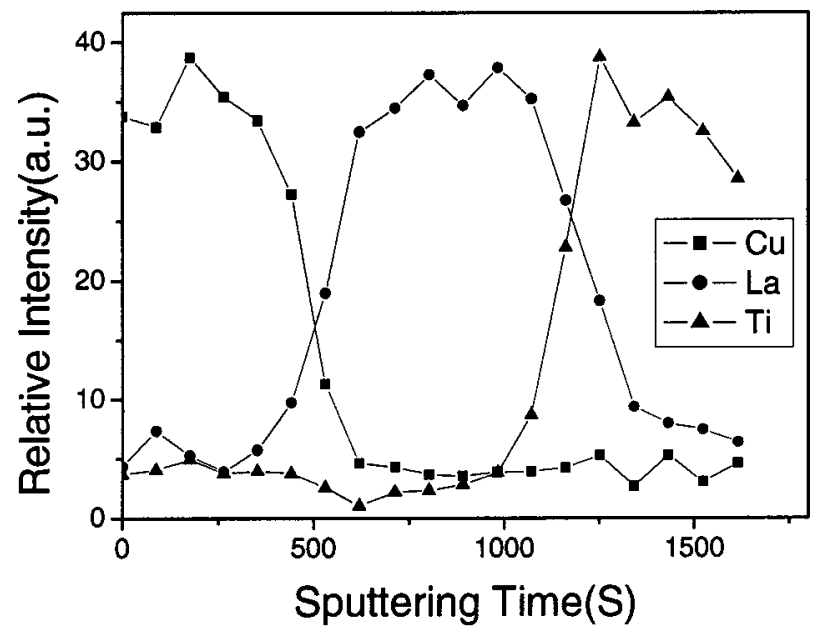

FIG. 3. The depth dependence of element $\mathrm{Cu}, \mathrm{La}$, and $\mathrm{Ti}$ composition in sample C.

$\mathrm{C}$ and E. Here the theoretical simulation is based on Fresnel's law. ${ }^{14}$ The corresponding simulation parameters are listed in Table II, from which one can see that there is a difference between the nominal and real thickness of the sublayers and transient layer existing at the YBCO/LCMO and LCMO/STO interfaces, with the thicknesses of about 3 and $2 \mathrm{~nm}$, respectively. The results of AES, shown in Fig. 3, confirmed that the transient layer is caused by the interdiffusion at the interface. In addition, the surface root-meansquare (rms) roughness for samples $\mathrm{C}$ and $\mathrm{E}$ is, respectively, 0.45 and $0.74 \mathrm{~nm}$, which is consistent with the observation from AFM within experimental error. From the above results, we can conclude that YBCO film, deposited on a LCMO template layer, is partially strained due to the lattice mismatch and the surface is flat in spite of the existence of a transient layer.

Figure 4 presents the temperature dependence of sample resistivity without magnetic field applied. Samples A and B are superconducting with the critical temperature $T_{c 0}$ (midpoint, $T_{c}$ ) at $83 \mathrm{~K}$. The critical current density at $77 \mathrm{~K}$ is estimated to be larger than $0.5 \times 10^{5} \mathrm{~A} / \mathrm{cm}^{2}$. The superconductivity is obviously depressed while comparing with those

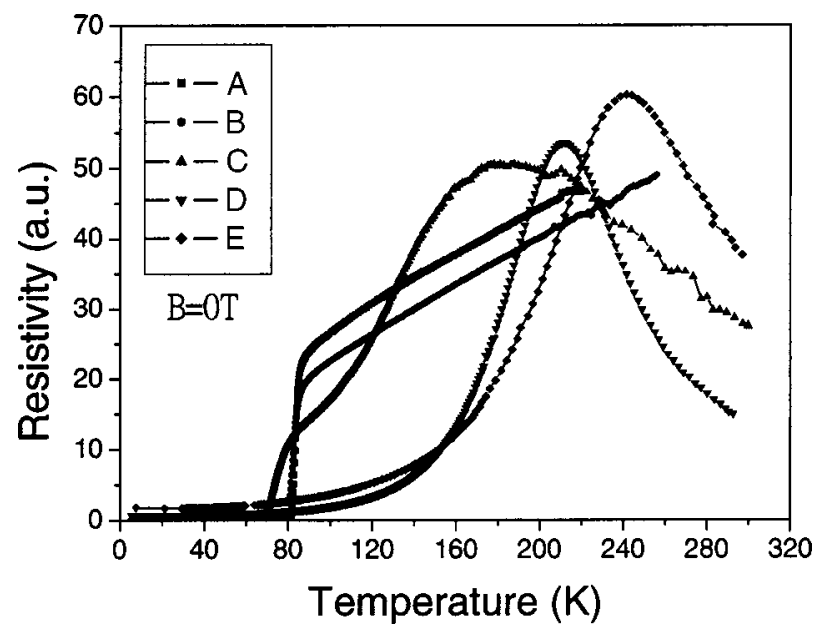

FIG. 4. The temperature dependence of resistance for samples without magnetic field applied. 
TABLE II. The simulation results of the grazing incident $\mathrm{x}$-ray reflectivity profiles.

\begin{tabular}{|c|c|c|c|c|}
\hline $\begin{array}{l}\text { Simulation } \\
\text { results }\end{array}$ & $\begin{array}{l}\text { Simulation } \\
\text { layers }\end{array}$ & $\begin{array}{c}\text { Simulation } \\
\text { thickness }( \pm 2 \AA)\end{array}$ & $\begin{array}{l}\text { Mass density } \\
\left(\mathrm{g} / \mathrm{cm}^{3}\right)\end{array}$ & $\begin{array}{c}\text { Surface and interface } \\
\text { roughness of layers } \\
( \pm 0.5 \AA)\end{array}$ \\
\hline \multirow{8}{*}{ Sample C } & Surf. & 19 & 3.43 & $\sigma_{\text {surf }}=4.5$ \\
\hline & YBCO & 134 & 6.27 & $\sigma_{\mathrm{YBCO}}=5.0$ \\
\hline & Transient & 33 & 6.19 & $\sigma_{\text {Trans } 2}=4.3$ \\
\hline & Layer2 & & & \\
\hline & LCMO & 308 & 6.51 & $\sigma_{\mathrm{LCMO}}=4.2$ \\
\hline & Transient & 22 & 6.21 & $\sigma_{\text {Trans } 1}=2.1$ \\
\hline & Layer1 & & & \\
\hline & STO & - & 5.12 & $\sigma_{\mathrm{LCMO} / \mathrm{STO}}=4.6$ \\
\hline \multirow{7}{*}{ Sample E } & Surf. & 12 & 3.98 & $\sigma_{\text {surf }}=7.4$ \\
\hline & YBCO & 48 & 6.23 & $\sigma_{\mathrm{YBCO}}=6.8$ \\
\hline & Transient & 22 & 6.09 & $\sigma_{\text {Trans } 2}=5.5$ \\
\hline & Layer2 & & & \\
\hline & LCMO & 327 & 6.44 & $\sigma_{\mathrm{LCMO}}=5.2$ \\
\hline & Transient & 16 & 5.98 & $\sigma_{\text {Trans } 1}=1.7$ \\
\hline & $\begin{array}{c}\text { Layer1 } \\
\text { STO }\end{array}$ & - & 5.12 & $\sigma_{\mathrm{LCMO}} \mathrm{STO}=4.8$ \\
\hline
\end{tabular}

YBCO films using other nonmagnetic buffers such as $\mathrm{Nd}_{2} \mathrm{CuO}_{4} \cdot{ }^{15}$ For samples $\mathrm{D}$ and $\mathrm{E}$, there only appears a resistivity maximum with $T_{M}$ at about 210 and $250 \mathrm{~K}$, respectively. It is well known that in the $R-T$ curve of perovskite manganite $T_{M}$ corresponds to the metal-insulator transition and it is a little lower than the ferromagnetic-paramagnetic transition temperature $T_{\text {Curie }}$. As for sample $\mathrm{C}$, the superconductivity is still observed, but $T_{c 0}$ has dropped to $78 \mathrm{~K}$. Sample $\mathrm{C}$ also shows resistance maximum with $T_{M}$ at about $170 \mathrm{~K}$ at the same time. This means that superconductivity and ferromagnetism perhaps coexist in sample $\mathrm{C}$ with the YBCO thickness of $12.5 \mathrm{~nm}$.

The transport properties described above clearly imply that superconductivity is competing with ferromagnetism in LCMO/YBCO bilayers. Considering the pair breaking effect enhanced by the spin-polarized carriers injection, the superconductivity will be suppressed because a certain thickness of YBCO will become "effectively" normal. Thereby the injection length is roughly estimated to be less than $25 \mathrm{~nm}$. This value is much less than the results reported in Ref. 13. It is still unclear whether the injection length is related to the LCMO thickness or not. Most interestingly, superconductivity and ferromagnetism coexist but are suppressed by each other in sample $\mathrm{C}$ with the $\mathrm{YBCO}$ thickness of $12.5 \mathrm{~nm}$. The YBCO thickness and thereby the strain state of bilayer is another factor for the transport properties of YBCO/LCMO heterostructures. As can be seen from Fig. 4, for samples C, $\mathrm{D}$, and $\mathrm{E}$, metal-insulator transition temperature $T_{M}$ is strongly influenced by the YBCO thickness and thereby the strain state of bilayers. In addition, the disorder at the interface may also be an influence factor that should not be negligible.

\section{CONCLUSIONS}

In summary, we prepared well (001)-oriented YBCO/ LCMO layered materials by magnetron sputtering. The mi- crostructures and transport properties were investigated. The results show that superconductivity and ferromagnetism perhaps coexist in the bilayer with the YBCO thickness of 12.5 $\mathrm{nm}$; while in the sample with YBCO thickness less than 8 $\mathrm{nm}$, only ferromagnetism was observed, with metalinsulator transition temperature $T_{M}$ strongly influenced by the strain state of bilayers.

\section{ACKNOWLEDGMENTS}

This work has been supported by the National Natural Science Foundation of China, the Provincial Natural Science Foundation of Jiangsu, the State Key Project of Fundamental Research, and the Chinese Ministry of Education.

${ }^{1}$ Z. Radovic, L. Dobrosavljevic-Grujic, A. I. Buzdin, and J. R. Clem, Phys. Rev. B 38, 2388 (1988).

${ }^{2}$ Z. Radovic, M. Ledvij, L. Dobrosavljevic-Grujic, A. I. Buzdin, and J. R. Clem, Phys. Rev. B 44, 759 (1988).

${ }^{3}$ C. A. R. Sa de Melo, Phys. Rev. Lett. 79, 1933 (1997).

${ }^{4}$ H. K. Wong and J. B. Ketterson, J. Low Temp. Phys. 63, 139 (1986).

${ }^{5}$ C. Strunk, C. Surgers, U. Paschen, and H. V. Lohneysen, Phys. Rev. B 49, 4053 (1994).

${ }^{6}$ L. Lazar, K. Westerholt, H. Zabel, L. R. Tagirov, Yu. V. Goryunov, N. N. Garif'yanov, and I. A. Garifullin, Phys. Rev. B 61, 3711 (2000), and references therein.

${ }^{7}$ K. Chahara, T. Ohno, M. Kasai, Y. Kanke, and Y. Kozono, Appl. Phys. Lett. 62, 780 (1993).

${ }^{8}$ G. Jakob, V. V. Moshchalkov, and Y. Bruynseraede, Appl. Phys. Lett. 66, 2564 (1995)

${ }^{9}$ Y. Inokuma, M. Kanai, and T. Kawai, Jpn. J. Appl. Phys., Part 1 36, 7169 (1997).

${ }^{10}$ P. Prieto et al., J. Appl. Phys. 89, 8026 (2001).

${ }^{11}$ Z. Sefrioui et al., http://xxx.lanl.gov/cond-mat/0207164.

${ }^{12}$ V. A. Vas'ko, V. A. Larkin, P. A. Kraus, K. R. Nikolaev, D. E. Grupp, C. A. Nordman, and A. M. Goldman, Phys. Rev. Lett. 78, 1134 (1997).

${ }^{13}$ S. P. Pai, S. Wanchoo, S. C. Purandare, T. Banerjee, P. R. Apte, A. M. Narsale, and R. Pinto, Pramana J. Phys. 58, 1147 (2002).

${ }^{14}$ L. G. Parrat, Phys. Rev. 95, 359 (1954).

${ }^{15}$ J. Gao, W. H. Tang, and T. C. Chui, Physica C 330, 33 (2000). 
Journal of Applied Physics is copyrighted by the American Institute of Physics (AIP). Redistribution of journal material is subject to the AIP online journal license and/or AIP copyright. For more information, see http://ojps.aip.org/japo/japcr/jsp

Copyright of Journal of Applied Physics is the property of American Institute of Physics and its content may not be copied or emailed to multiple sites or posted to a listserv without the copyright holder's express written permission. However, users may print, download, or email articles for individual use. 